\title{
Anisotropic Behavior of Cosmological Models with Exponential and Hyperbolic Scale Factors
}

\author{
Fakhereh Md. Esmaeili \\ School of Physics, University of Hyderabad, Hyderabad, India \\ Email: astrosat92@gmail.com
}

How to cite this paper: Esmaeili, F.M. (2018) Anisotropic Behavior of Cosmological Models with Exponential and Hyperbolic Scale Factors. Journal of High Energy Physics, Gravitation and Cosmology, 4, 223-235.

https://doi.org/10.4236/jhepgc.2018.42017

Received: February 14, 2018

Accepted: March 17, 2018

Published: March 20, 2018

Copyright $\odot 2018$ by author and Scientific Research Publishing Inc. This work is licensed under the Creative Commons Attribution International License (CC BY 4.0).

http://creativecommons.org/licenses/by/4.0/

\section{Abstract}

In this paper, the cosmological models of the universe are constructed in $f(R, T)$ gravity with choice of the functional $f(R, T)$ in the form $f_{1}(R)=\lambda R$ and $f_{2}(T)=\lambda T$. The space-time considered here is Bianchi Type I and the energy momentum tensor is in the form of perfect fluid. Two cosmological models are presented using a power form of exponential function and a hyperbolic form. The energy conditions along with the state finder diagnostic pair have been obtained and analyzed.

\section{Keywords}

$f(R, T)$ Cosmology, Hyperbolic Scale Factor, Bianchi Type I, Hubble Parameter

\section{Introduction}

Modified theory of gravity is one of the prominent alternative theories to Einstein's general theory of relativity (GR). Though GR is very much known for its excellent success, it is silent on certain specific topics on the study of the universe. One of such topics is the late time cosmic acceleration. It has been learnt that modified theory of gravity has been successful in describing the late time cosmic acceleration and accelerated expansion of the universe. Thus, modified gravity nowadays is a topic of great interest. Out of all prospective variants of modified gravity, the $f(R, T)$ gravity theory proposed by Harko et al. [1] is one of the most fascinating theories. In this theory, they have generalized the basic $f(R)$ theory by taking the gravitational Lagrangian as a random function of Ricci scalar $R$ and trace of the stress-energy tensor $T$. In this theory, the action for a polynomial function of Ricci scalar is defined as the Einstein-Hilbert action that leads to obtain the Einstein field equation according 
to principle of least action. Second, different types of operation such as variation of action were placed. Considering the universe was filled by perfect fluid that the stress energy-momentum tensor of the matter is shown as $T_{\mu \nu}$, the action for $f(R, T)$ can be described as

$$
S=\frac{1}{16 \pi G} \int \sqrt{-g} f(R, T) \mathrm{d}^{4} x+\int L_{m} \sqrt{-g} \mathrm{~d}^{4} x
$$

After some manipulation on variation of action and assuming the relation $\theta_{\mu v}=-2 T_{\mu v}-p g_{\mu v}$, the field equations for $f(R, T)$ gravity can be obtained as

$$
f_{R} R_{\mu \nu}-\frac{1}{2} f(R, T) g_{\mu \nu}+\left(g_{\mu v} \square-\nabla_{\mu} \nabla_{v}\right) f_{R}=8 \pi T_{\mu \nu}-f_{T} T_{\mu v}-f_{T} \theta_{\mu \nu}
$$

where the functional $f(R, T)$ is the function of Ricci scalar $R$ and stress-energy momentum tensor $T, f_{R}$ and $f_{T}$ respectively denote the partial derivative of $f(R, T)$ with respect to $R$ and $T$ respectively.

Several aspects of $f(R, T)$ gravity have been investigated so far: Yousaf et al. [2] have investigated causes of irregular energy density in $f(R, T)$ gravity. Alves et al. [3] have studied the gravitational waves in $f(R, T)$ gravity. It can also be noted that, the recent starting of the gravitational wave astronomy through various detections by LIGO (Laser Interferometer Gravitational-Wave Observatory) will be useful in discriminating between Einstein's General Theory of Relativity and the extended theories of gravity [4] [5]. Sharif and Zubair [6] have discussed the equilibrium picture of thermodynamics at the apparent horizon of FRW universe whereas Zaregonbadi et al. [7] obtained the dark matter solution from $f(R, T)$ gravity. Mishra et al. [8] have studied the dynamical behaviour of the $f(R, T)$ gravity. Also, Mishra and Vadrevu [9] have obtained the exact solution in cylindrically symmetric space-time. Agrawal and Pawar [10] obtained the magnetized domain wall in $f(R, T)$ gravity in a Bianchi type V space-time. Chakraborty [11] has suggested that a part of the arbitrary function $f(R, T)$ can be determined if the conservation of stress-energy tensor is taken into consideration in the original version [1]. Several authors have studied different aspects of viscous fluid in modified theory of gravity [12] [13] [14] [15] [16]. Shamir and Kanwal [17] studied the anisotropic universe using Noether symmetries in locally rotationally symmetric Bianchi type I whereas Mishra et al. [18] reconstructed the anisotropic universe in Bianchi type $\mathrm{V}$ space-time. The anisotropic behaviour of the cosmological models has been investigated by many authors [19] [20] [21] [22] [23].

We intend to assume the functional form of $f(R, T)$ in such a way that it can be reduced to the usual field equations in GR. A popular choice is $f(R, T)=\lambda R+\lambda T$, where $\lambda$ is constant. So, the field equation in modified theory of gravity can be reduced to

$$
R_{\mu v}-\frac{1}{2} R g_{\mu v}=\left(\frac{8 \pi}{\lambda}+1\right) T_{\mu v}+\Lambda g_{\mu v}
$$

where $\Lambda=\frac{1}{2}(\rho-p)$ is the Effective Cosmological Constant henceforth 
abbreviated as ECC.

\section{Basic Formalism of the Model}

We have considered Bianchi type I space-time in the form

$$
\mathrm{d} s^{2}=\mathrm{d} t^{2}-A^{2} \mathrm{~d} x^{2}-B^{2}\left(\mathrm{~d} y^{2}+\mathrm{d} z^{2}\right)
$$

The metric potentials $A$ and $B$ are function of cosmic time only. The energy-momentum tensor for the perfect fluid can be expressed as

$$
T_{\mu v}=(p+\rho) u_{\mu} u_{v}-p g_{\mu v}
$$

whereas $u^{\mu}$ is the four velocity vector of the fluid. Now, the field Equation (3), for the space-time (4) with the energy momentum tensor (5) can be obtained as

$$
\begin{gathered}
2 \frac{\ddot{B}}{B}+\frac{\dot{B}^{2}}{B^{2}}=\frac{1}{2} \rho-\alpha p \\
\frac{\dot{A} \dot{B}}{A B}+\frac{\ddot{B}}{B}+\frac{\ddot{A}}{A}=\frac{1}{2} \rho-\alpha p \\
2 \frac{\dot{A} \dot{B}}{A B}+\frac{\dot{B}^{2}}{B^{2}}=\alpha \rho-\frac{1}{2} p
\end{gathered}
$$

where $\alpha=\frac{8 \pi}{\lambda}+\frac{3}{2}$ and dot represents the derivative with respect to the cosmic time. Equations (6)-(8) are having four knowns viz.: $A, B, p, \rho$. In view of this with an intention to obtain a solution to the field equation, we have transformed all the field Equations (6)-(8) in terms of Hubble rate. The directional Hubble rates can be considered as $H_{x}=\frac{\dot{A}}{A}, H_{y}=\frac{\dot{B}}{B}=\frac{\dot{C}}{C}$. Now, the set of field Equations (6)-(8) can be reduced to

$$
\begin{gathered}
2 \dot{H}_{y}+3 H_{y}^{2}=\frac{1}{2} \rho-\alpha p \\
H_{x} H_{y}+\dot{H}_{y}+\dot{H}_{x}+H_{x}^{2}+H_{y}^{2}=\frac{1}{2} \rho-\alpha p \\
2 H_{x} H_{y}+H_{y}^{2}=\alpha \rho-\frac{1}{2} p
\end{gathered}
$$

Now, with suitable algebraic manipulations the pressure and energy density with respect to Hubble term can be obtained as

$$
\begin{aligned}
& p=\frac{1}{\frac{1}{4}-\alpha^{2}}\left[\left(3 \alpha-\frac{1}{2}\right) H_{y}^{2}+2 \alpha \dot{H}_{y}-H_{x} H_{y}\right] \\
& \rho=\frac{1}{\frac{1}{4}-\alpha^{2}}\left[\left(\frac{3}{2}-\alpha\right) H_{y}^{2}+\dot{H}_{y}-2 \alpha H_{x} H_{y}\right]
\end{aligned}
$$

Then, we can define the Equation of State (EoS) Parameter and ECC in Hubble term as 


$$
\begin{gathered}
\omega=\frac{p}{\rho}=\frac{(6 \alpha-1) H_{y}^{2}+4 \alpha \dot{H}_{y}-2 H_{x} H_{y}}{(3-2 \alpha) H_{y}^{2}+2 \dot{H}_{y}-4 \alpha H_{x} H_{y}} \\
\Lambda=\frac{\rho-p}{2}=\frac{2\left(2 H_{y}^{2}+\dot{H}_{y}+H_{x} H_{y}\right)}{(1+2 \alpha)}
\end{gathered}
$$

We can study the dynamical features of the model, which would be decided by the physical quantities given in (12)-(15). However all these quantities depend on the Hubble parameter which is subsequently depends on the mean scale factor. So, if we understand the behaviour of mean scale factor, the background cosmology and the associated dynamics can be studied.

\section{Dynamical Features of the Models}

Different scale factors has different roles in the study of cosmic dynamics of the universe. For instance: power law cosmology emerged as an alternative to $\Lambda \mathrm{CDM}$ model; the cosmic dynamics dominated by the exponential scale factor at late phase; and the hybrid scale factor produces a cosmic transit from early deceleration to late time cosmic acceleration. However not many research has been done on hyperbolic scale factor and fractional power of an exponential functions to understand the cosmic dynamics of the universe. Therefore, we have considered two scale factors such $\zeta \tanh (\eta t)$ and $\left(\mathrm{e}^{n \zeta t}-1\right)^{\frac{1}{n}}$, where $\eta$ and $\zeta$ both are constants.

\subsection{Case-I}

In this case, we have considered the hyperbolic scale factor in the form $\mathcal{R}=\zeta \tanh (\eta t)$. Subsequently the Hubble parameter and deceleration parameter can be obtained as $H=\frac{\eta}{\cosh (\eta t) \sinh (\eta t)}$ and $q=2 \sinh ^{2}(\eta t)$ respectively. A linear anisotropic relation among the directional Hubble rates is assumed i.e. $H_{x}=k H_{y}$, where $k$ is a constant. Hence, from Equations ((12), (13)), the pressure and energy density can be obtained as

$$
\begin{aligned}
& p=\frac{36 \eta^{2}}{\left(1-4 \alpha^{2}\right)(k+2) \cosh ^{2}(\eta t) \sinh ^{2}(\eta t)}\left[3 \alpha-\frac{1}{2}-\frac{2}{3} \alpha \cosh (2 \eta t)(k+2)-k\right](16) \\
& \rho=\frac{36 \eta^{2}}{\left(1-4 \alpha^{2}\right)(k+2) \cosh ^{2}(\eta t) \sinh ^{2}(\eta t)}\left[\frac{3}{2}-\alpha-\frac{1}{3} \cosh (2 \eta t)(k+2)-2 \alpha k\right]
\end{aligned}
$$

The graphical behaviour of pressure and energy density has been represented respectively in Figure 1 and Figure 2. It has been observed that the pressure starts from a high negative value and subsequently settled near a small value. At the same time, the energy density starts from a high positive value and at late times reduces to a small value, however in the entire evolution it remains in the positive domain only. It can be noted that the scaling constant $\lambda$ is also 


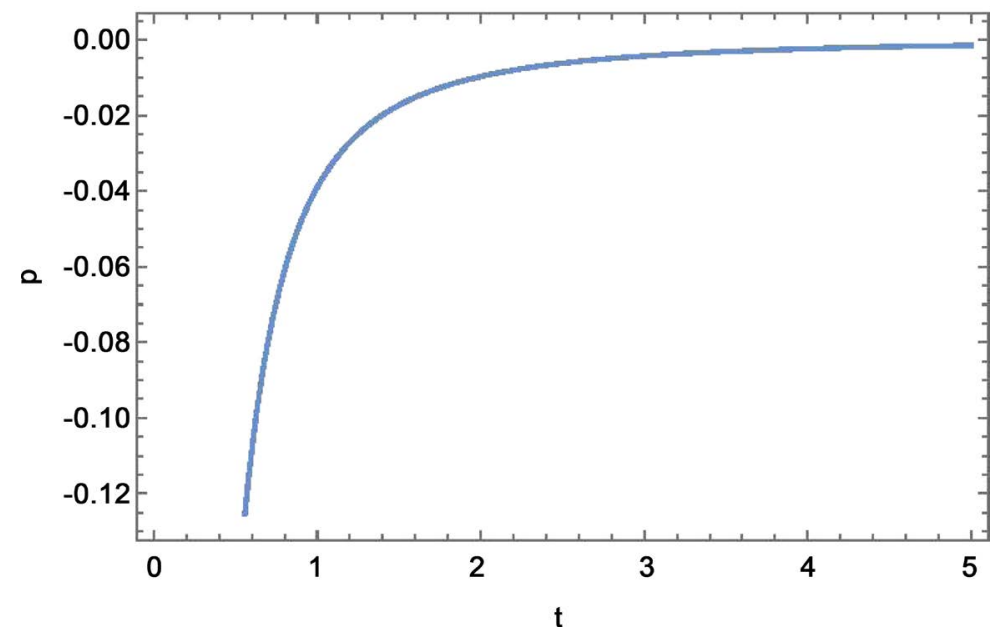

Figure 1. Graphical behaviour of $p$ versus $t(\eta=0.01, k=0.45, \lambda=0.5)$.

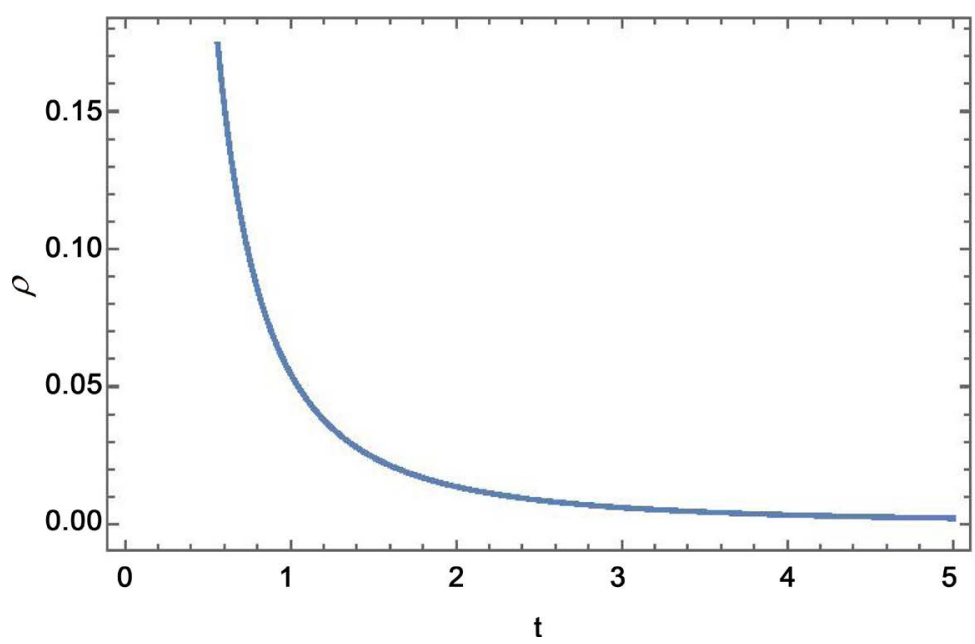

Figure 2. Graphical behaviour of $\rho$ versus $t(\eta=0.01, k=0.45, \lambda=0.5)$.

instrumental in changing the behaviour of both the pressure and energy density.

The EoS parameter ECC can be reduced from Equation (14) with the hyperbolic scale factor as

$$
\begin{gathered}
w=\frac{p}{\rho}=\frac{3 \alpha-\frac{1}{2}-\frac{2}{3} \alpha \cosh (2 \eta t)(k+2)-k}{\frac{3}{2}-\alpha-\frac{1}{3} \cosh (2 \eta t)(k+2)-2 \alpha k} \\
\Lambda=\frac{18 \eta^{2}}{\left(1-4 \alpha^{2}\right)(k+2) \cosh ^{2}(\eta t) \sinh ^{2}(\eta t)}\left[(1-2 \alpha)+\left(\frac{2}{3} \alpha-3\right) \cosh (2 \eta t)\right]
\end{gathered}
$$

The graphical behaviour of the EoS parameter and ECC respectively represented in Figure 3 and Figure 4. The EoS parameter starts evolving with a value $\approx-0.715$ and gradually increases and remains in the quintessence region, which satisfies the observational result. The ECC entirely stay in the positive domain and gradually decreases with increase in time which indicates that dominance of energy density. Even though, we have considered an hyperbolic 


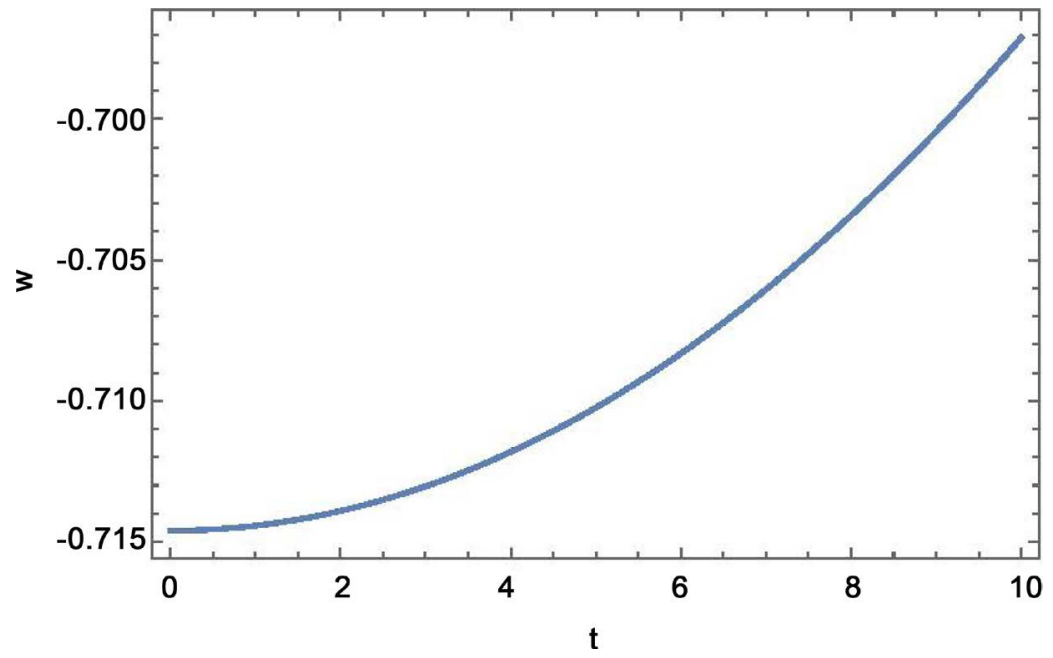

Figure 3. Graphical behaviour of $w$ versus $t(\eta=0.01, k=0.45, \lambda=0.5)$.

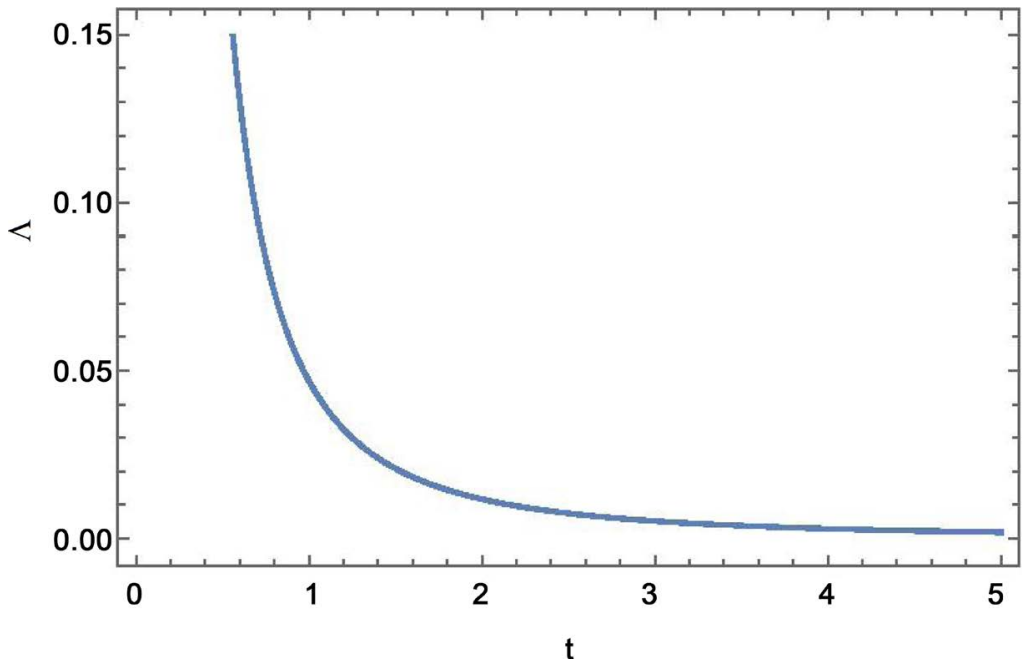

Figure 4. Graphical behaviour of $\Lambda$ versus $t(\eta=0.01, k=0.45, \lambda=0.5)$.

scale function which is always positive, still then the EoS parameter remain entirely in the acceptable range. So, it can be inferred that the role of the scaling constant is prominent on the expanding universe.

\subsection{Case-II}

In order to have a better understanding on the anisotropic universe, in this case, we have considered the scale factor in the power form of as a fraction of exponential function in the form $R=\left(\mathrm{e}^{n \zeta t}-1\right)^{\frac{1}{n}}$. It can be noted that if any of the constant vanishes, the scale factor becomes unity. Subsequently the Hubble parameter and deceleration parameter can be obtained as $H=\frac{3 n \zeta \mathrm{e}^{n \zeta t}}{\mathrm{e}^{n \zeta t}-1}$ and $q(t)=-1+\frac{n}{\mathrm{e}^{n \zeta t}}$. The pressure and energy density of the model can be obtained from Equations ((12), (13)), 


$$
\begin{aligned}
& p=\left[\frac{1}{\frac{1}{4}-\alpha^{2}}\right]\left[\frac{9 \zeta^{2} \mathrm{e}^{2 n \zeta t}}{(k+2)^{2}\left(\mathrm{e}^{n \zeta t}-1\right)^{2}}\right]\left[3 \alpha-\frac{1}{2}-\frac{2 n \alpha}{3}(k+2) \mathrm{e}^{-n \zeta t}-k\right] \\
& \rho=\left[\frac{1}{\frac{1}{4}-\alpha^{2}}\right]\left[\frac{9 \zeta^{2} \mathrm{e}^{2 n \zeta t}}{(k+2)^{2}\left(\mathrm{e}^{n \zeta t}-1\right)^{2}}\right]\left[\frac{3}{2}-\alpha-\frac{n}{3}(k+2) \mathrm{e}^{-n \zeta t}-2 \alpha k\right]
\end{aligned}
$$

The pressure increases from some large negative value $\approx-0.8$ at an initial stage and slowly vanishing to small values at late times. It is felt that the choice of scaling parameter affects substantially the magnitude of the pressure (Figure 5). On the other hand, the energy density always remain in the positive domain and decreases to small value at late times (Figure 6).

The EoS parameter and ECC can be obtained as

$$
\begin{gathered}
w=\frac{3 \alpha-\frac{1}{2}-\frac{2 n \alpha}{3}(k+2) \mathrm{e}^{-n \zeta t}-k}{\frac{3}{2}-\alpha-\frac{n}{3}(k+2) \mathrm{e}^{-n \zeta t}-2 \alpha k} \\
\Lambda=\frac{9 \zeta^{2} \mathrm{e}^{2 n \zeta t}(1-2 \alpha)\left(1-\frac{n \mathrm{e}^{-n \zeta t}}{3}\right)}{2\left(\frac{1}{4}-\alpha^{2}\right)\left(\mathrm{e}^{n \zeta t}-1\right)^{2}(k+2)}
\end{gathered}
$$

It is observed that the EoS parameter as represented in Figure 7 starts from a value $\approx-0.95$ at initial time and as time increases the value of the parameter increases. However, it remains in the negative domain and stay in the quintessence region as suggested observationally. At the same time, the ECC (Figure 8 ) remains entirely in the positive domain and gradually decreases and remain idle at the late time of the evolution.

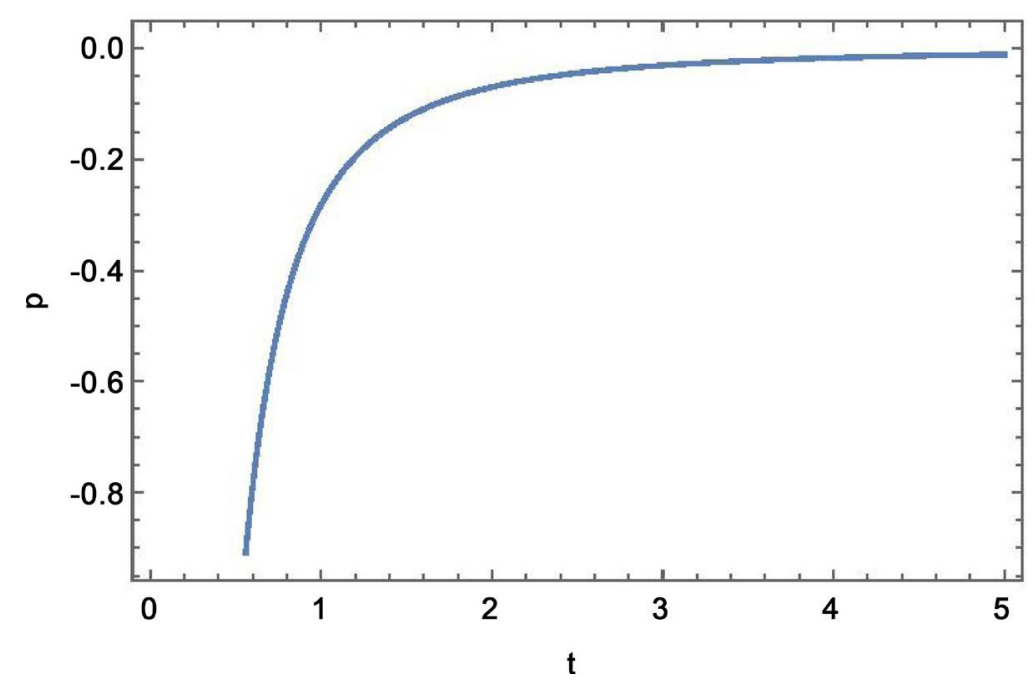

Figure 5. Graphical behaviour of $p$ versus $t(\zeta=-0.02, n=0.5, k=0.62, \lambda=0.68)$. 


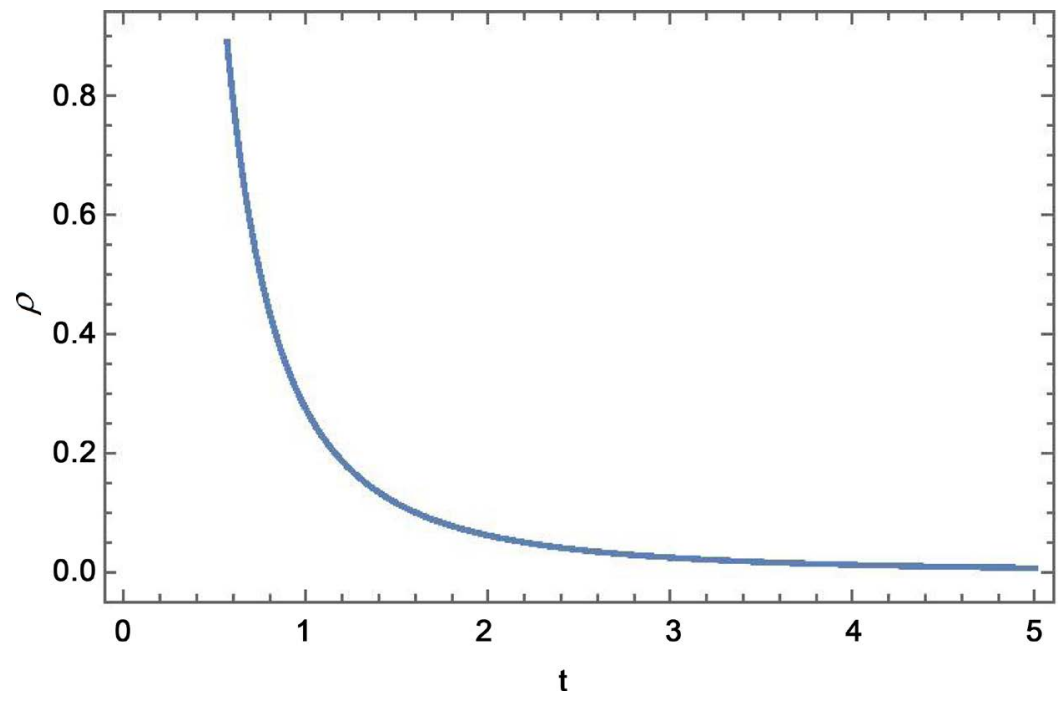

Figure 6. Graphical behaviour of $\rho$ versus $t(\zeta=-0.02, n=0.5, k=0.62, \lambda=0.68)$.

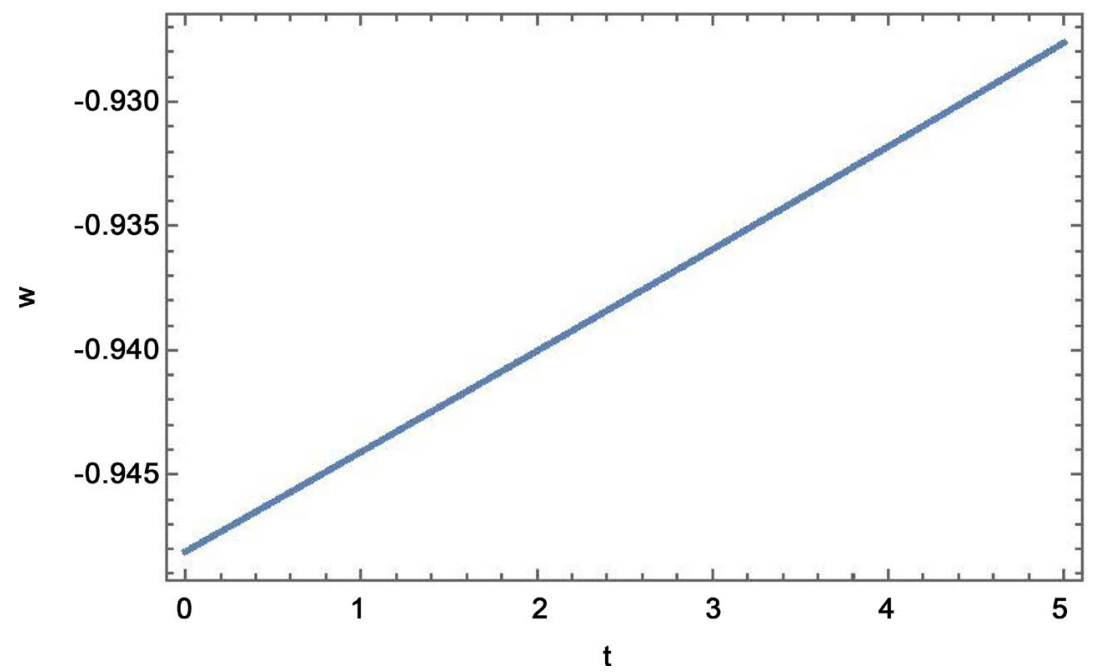

Figure 7. Graphical behaviour of $w$ versus $t(\zeta=-0.02, n=0.5, k=0.62, \lambda=0.68)$.

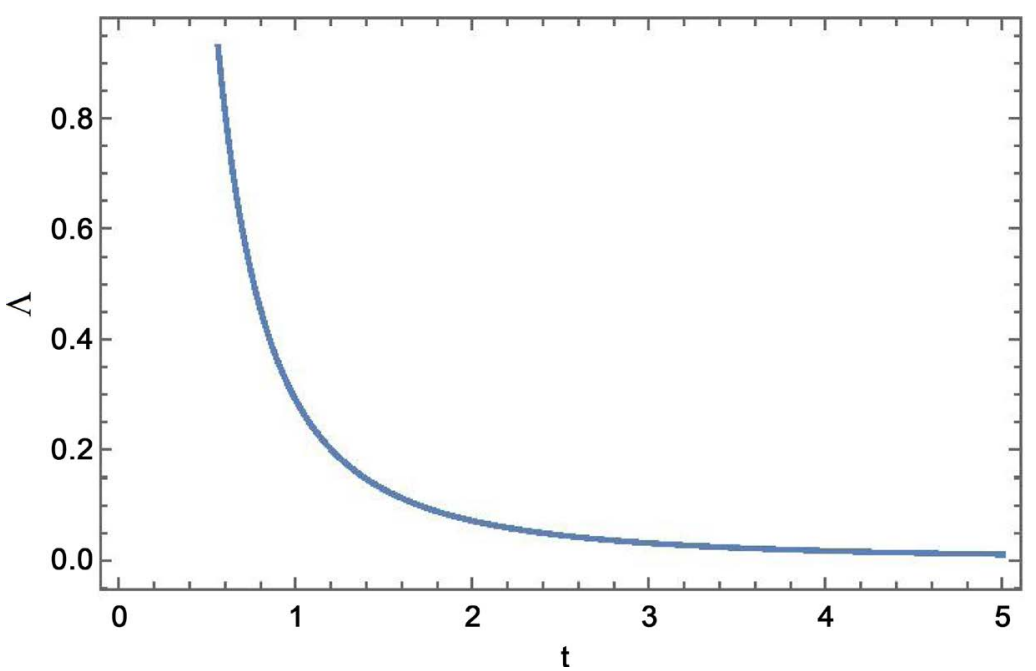

Figure 8. Graphical behaviour of $\Lambda$ versus $t(\zeta=-0.02, n=0.5, k=0.62, \lambda=0.68)$. 


\section{Physical Parameters of the Model}

The state finder diagnostic pair shows the geometrical behaviour of the model. The acceptability of the model depends on the $(r, s)$ diagnosis that compares with the $\Lambda_{\mathrm{CDM}}$ model. For Case-I, the pair can be calculated as

$$
\begin{gathered}
r=\sinh ^{2}(\eta t)\left(1-2 \sinh ^{2}(\eta t)\right) \\
s=\frac{\sinh ^{2}(\eta t)\left(1-2 \sinh ^{2}(\eta t)\right)-1}{6 \sinh (\eta t)-\frac{3}{2}}
\end{gathered}
$$

Similarly for case II, the pair can be obtained as

$$
\begin{aligned}
& r=\frac{\mathrm{e}^{n \zeta t}+(n-3) n+n^{2} \mathrm{e}^{-n \zeta t}}{\mathrm{e}^{2 \zeta n t}} \\
& s=\frac{\mathrm{e}^{n \zeta t}\left(1+n^{2}\right)+n^{2}-3 n-1}{3\left(\frac{n-\mathrm{e}^{n \zeta t}}{\mathrm{e}^{n \zeta t}}-\frac{1}{2}\right)}
\end{aligned}
$$

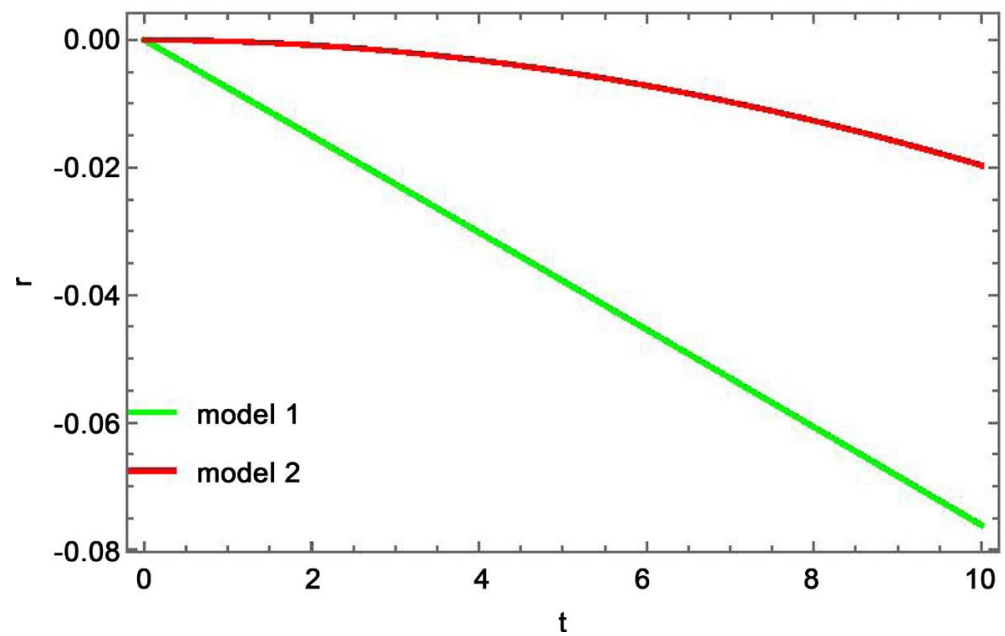

Figure 9. Graphical behaviour of $r$ versus $t$.

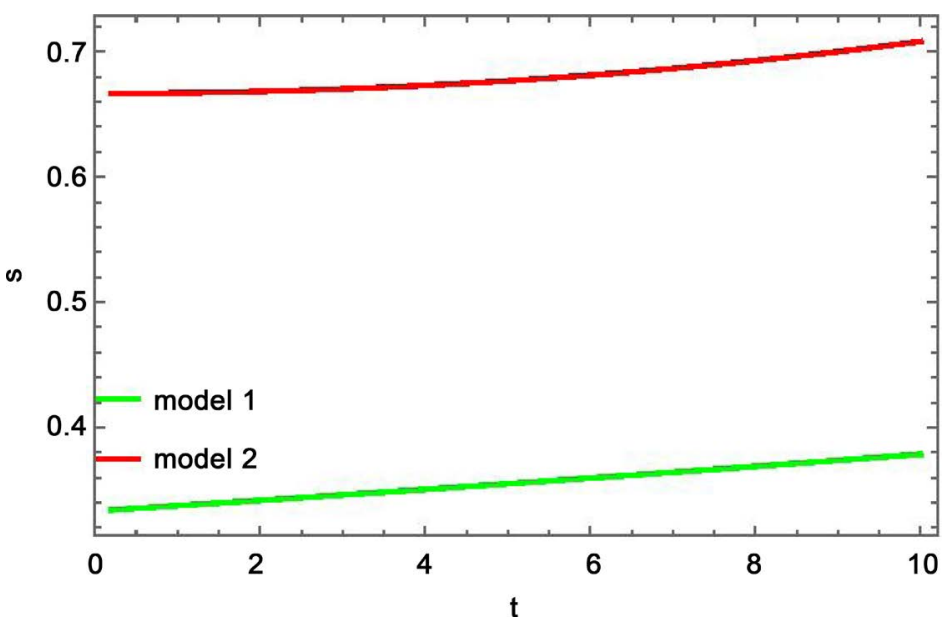

Figure 10. Graphical behaviour of $s$ versus $t$. 
From the graphs Figure 9 and Figure 10, where the Green line gives the behaviour of the parameter for first model and Red line for second model. For both the models we can infer that the parameter $r$ decreases with increase in time whereas the parameter $s$ increases. With a suitable substitution of the constants the pair can be reduced to $(r, s) \approx(1,0)$.

The Energy conditions put some additional constraints to the model. For a perfect fluid the energy conditions can be defined as: Null Energy Condition (NEC), $\rho+p \geq 0$, Weak Energy Condition (WEC), $\rho+p \geq 0$, Strong Energy Condition (SEC) $\rho+3 p \geq 0$, Dominant Energy Condition(DEC), $\rho \pm p \geq 0$. For Case-I the energy conditions can be calculated as

$$
\begin{aligned}
p+\rho= & \frac{9 \eta^{2}}{\left(\frac{1}{4}-\alpha^{2}\right)(k+2)^{2} \sinh ^{2}(\eta t) \cosh ^{2}(\eta t)} \\
& \times\left[(1+2 \alpha)(1-k)-(k+2)\left(3+\frac{2}{3} \alpha\right) \cosh (2 \eta t)\right] \\
\rho+3 p= & \frac{9 \eta^{2}}{\left(\frac{1}{4}-\alpha^{2}\right)(k+2)^{2} \sinh ^{2}(\eta t) \cosh ^{2}(\eta t)} \\
& \times\left[8 \alpha-k(3+2 \alpha)-\cosh ^{2}(2 \eta t)(k+2)(3+2 \alpha)\right] \\
\rho- & p=\frac{9 \eta^{2}}{\left(\frac{1}{4}-\alpha^{2}\right)(k+2)^{2} \sinh ^{2}(\eta t) \cosh ^{2}(\eta t)} \\
& \quad \times\left[(k+2)\left(1-2 \alpha-\cosh ^{2}(2 \eta t)\left(3+\frac{2}{3} \alpha\right)\right)\right]
\end{aligned}
$$

Similarly, for Case-II, the energy conditions can be obtained as

$$
\begin{gathered}
\rho+p=\frac{9 \zeta^{2} \mathrm{e}^{2 n \zeta t}}{\left(\frac{1}{4}-\alpha^{2}\right)(k+2)^{2}\left(\mathrm{e}^{n \zeta t}-1\right)^{2}}(1+2 \alpha)\left[1-k-\frac{n}{3}(k+2) \mathrm{e}^{-n \zeta t}\right] \\
\rho+3 p=\frac{9 \zeta^{2} \mathrm{e}^{2 n \zeta t}}{\left(\frac{1}{4}-\alpha^{2}\right)(k+2)^{2}\left(\mathrm{e}^{n \zeta t}-1\right)^{2}}\left[8 \alpha-k(3+2 \alpha)-n(k+2) \mathrm{e}^{-n \zeta t}\left(\frac{1}{3}+2 \alpha\right)\right] \\
\rho-p=\frac{9 \zeta^{2} \mathrm{e}^{2 n \zeta t}}{\left(\frac{1}{4}-\alpha^{2}\right)(k+2)^{2}\left(\mathrm{e}^{n \zeta t}-1\right)^{2}}(1-2 \alpha)\left[1-\frac{n}{3} \mathrm{e}^{-n \zeta t}\right]
\end{gathered}
$$

The graphical behaviour of the energy conditions for Case-I and Case-II are represented respectively in Figure 11 and Figure 12 for representative values of the parameters. The energy conditions in Figure 11 are calculated for $\eta=0.01$, $\lambda=0.5$ and in Figure 12 the values are $n=0.5, \lambda=0.68, \zeta=-0.02$. The Green $(\mathrm{Q})$, Orange $(\mathrm{U})$, Blue $(\mathrm{G})$ phase in both the graphs respectively represent the behaviour for WEC, SEC and DEC. We have explored the effect of 


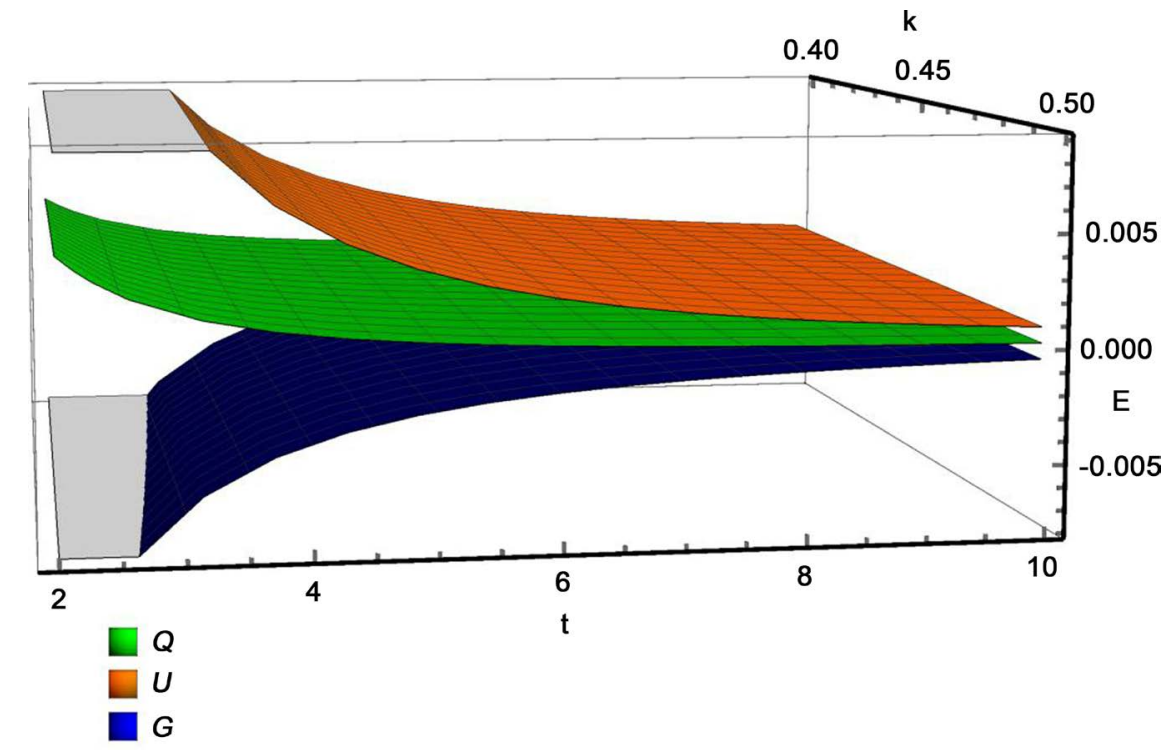

Figure 11. Graphical behaviour of $Q, U, G$ versus $t$.

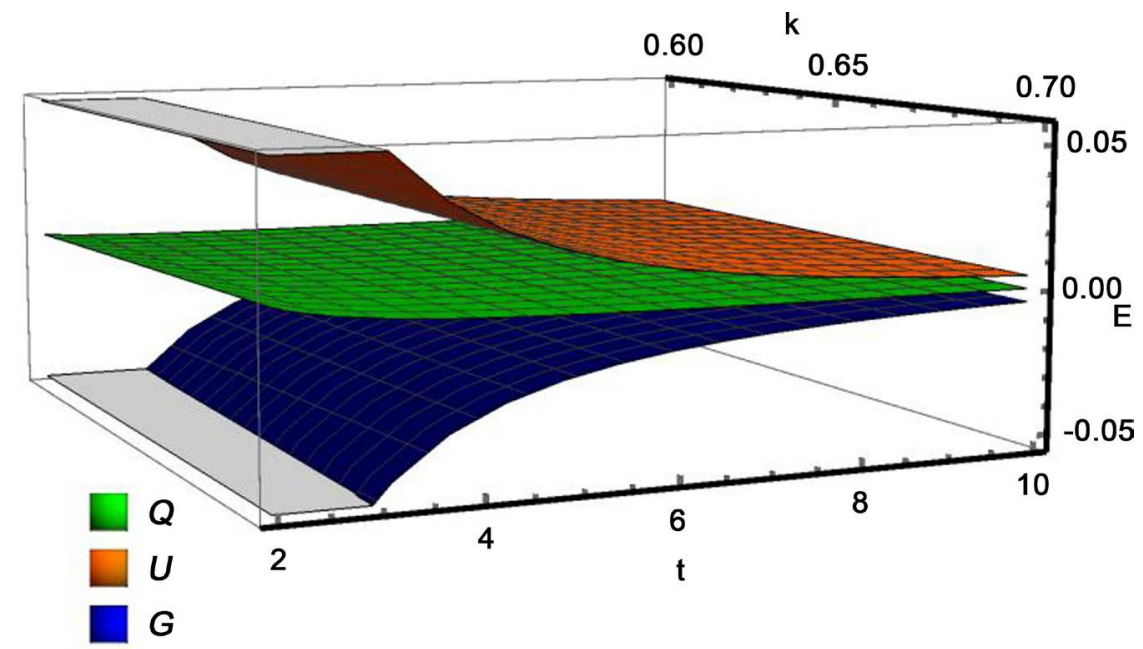

Figure 12. Graphical behaviour of $Q, U, G$ versus $t$.

anisotropy on the energy conditions. Here the anisotropy parameter $k$ is taken as a free parameter. The behaviour of the energy conditions remain the same for different choices of the scaling constant $\lambda$. Since we are interested in modified gravity models close to GR, we assume a small value of the scaling constant $\lambda$, the behaviour of the energy condition remains almost same.

The scalar expansion for the first model is $\theta=\frac{3 \eta}{\cosh (\eta t) \sinh (\eta t)}$ whereas for the second model $\theta=\frac{3 n \zeta \mathrm{e}^{n \zeta t}}{\mathrm{e}^{n \zeta t}-1}$. It can be noted that for both the models when $t \rightarrow 0, \theta \rightarrow \infty$ and $t \rightarrow \infty, \theta \rightarrow 0$. It indicates that the scalar expansion property supports the accelerated expansion of the universe. The rate of anisotropy for the first model is $\mathcal{A}=4\left(\frac{k-1}{k+2}\right)^{2}$ and for the second model is 
$\mathcal{A}=2\left(\frac{k-1}{k+2}\right)^{2}$. It is found that for $k=1$, the rate of anisotropy vanishes and confirms the isotropic nature of the space-time.

\section{Conclusion}

In this paper, we have studied the anisotropic behaviour of the cosmological model constructed with a Bianchi type I space-time and two different scale factors. In both the models, the state finder diagnostic pair is in the accepted value. The effect of the anisotropic parameters $k$ is investigated. The ratio of anisotropy reduces to zero when the anisotropic parameters in both the cases are unity. The behaviour of energy conditions remain same irrespective of the value of the scaling constant. The state finder diagnostic pair found to be in accordance with the present observed value. It is also concluded that an increase in cosmic anisotropy value in this work substantially affects the energy conditions.

\section{Acknowledgements}

The author is very much thankful to Prof. P. K. Suresh for his valuable suggestions during the preparation of the paper.

\section{References}

[1] Harko, T., Lobo, F.S.N., Nojiri, S. and Odintsov, S.D. (2011) $f(R, T)$ Gravity. Physical Review D, 84, Article ID: 024020. https://doi.org/10.1103/PhysRevD.84.024020

[2] Yousaf, Z., Bamba, K. and Bhatti, M.Z. (2016) Causes of Irregular Energy Density in $f(R, T)$ Gravity. Physical Review D, 93, Article ID: 124048. https://doi.org/10.1103/PhysRevD.93.124048

[3] Alves, M.E.S. Moraes, P.H.R.S., De Araujo, J.C.N. and Malheiro, M. (2016) Gravitational Waves in the $f(R, T)$ and $f\left(R, T^{\phi}\right)$ Theories of Gravity. Physical Review $D$, 94, Article ID: 024032. https://doi.org/10.1103/PhysRevD.94.024032

[4] Corda, C. (2018) Interferometric Detection of Gravitational Waves: The Definitive Test for General Relativity. International Journal of Modern Physics D, 18, 2275. https://doi.org/10.1142/S0218271809015904

[5] Corda, C. (2018) The Future of Gravitational Theories in the Era of the Gravitational Wave Astronomy. International Journal of Modern Physics D, 27, Article ID: 1850060

[6] Sharif, M. and Zubair, M. (2012) Thermodynamics in $f(R, T)$ Theory of Gravity. J.Cosmol, Astropart. Phys, 28.

[7] Zaregonbadi, R., Farhoudi, M. and Riazi, N. (2016) Dark Matter from $f(R, T)$ Gravity. Physical Review D, 94, Article ID: 084052. https://doi.org/10.1103/PhysRevD.94.084052

[8] Mishra, B., Tarai, S. and Tripathy, S.K. (2016) Dynamics of an Anisotropic Universe in $f(R, T)$ Theory. Advances in High Energy Physics, 1, 8543560.

[9] Mishra, B. and Vadrevu, S. (2017) Cylindrically Symmetric Cosmological Model of the Universe in Modified Gravity. Astrophysics and Space Science, 362, 26. 
[10] Agrawal, P. and Pawar, D.D. (2017) Magnetized Domain Wall in $f(R, T)$ Theory of Gravity. New Astronomy, 54, 56-60. https://doi.org/10.1016/j.newast.2017.01.006

[11] Chakraborty, S. (2013) An Alternative $f(R, T)$ Gravity Theory and the Dark Energy Problem. General Relativity and Gravitation, 45, 2039-2052. https://doi.org/10.1007/s10714-013-1577-y

[12] Mahanta, K.L. (2014) Bulk Viscous Cosmological Models in $f(R, T)$ Theory of Gravity. Astrophysics and Space Science, 353, 683-689. https://doi.org/10.1007/s10509-014-2040-6

[13] Ram, S. and Kumari, P. (2014) Bianchi Types I and V Bulk Viscous Fluid Cosmological Models in $f(R, T)$ Gravity Theory. Central European Journal of Physics, 12, 744-754.

[14] Chaturvedi, B. and Gupta, B.K. (2017) Six-Dimensional Bulk Viscous Fluid Cosmological Model in $f(R, T)$ Gravity Theory. Bulgarian Journal of Physics, 44, 288-298.

[15] Aygün, S. (2017) Marder Type Universe with Bulk Viscous String Cosmological Model in $f(R, T)$ Gravity. Turkish Journal of Physics, 41, 436-446.

https://doi.org/10.3906/fiz-1704-14

[16] Mishra, B., Tarai, S. and Pacif, S.K.J. (2018) Dynamics of Bianchi $V I_{h}$ Universe with Bulk Viscous Fluid in Modified Gravity. International Journal of Geometric Methods in Modern Physics, 15, 1850036. https://doi.org/10.1142/S0219887818500366

[17] Shamir, M.F. and Kanwal, F. (2017) Noether Symmetry Analysis of Anisotropic Universe in Modified Gravity. The European Physical Journal C, 77, 286. https://doi.org/10.1140/epjc/s10052-017-4869-7

[18] Mishra, B., Tarai, S. and Tripathy, S.K. (2017) Anisotropic Cosmological Reconstruction in $f(R, T)$ Gravity. arXiv:1709.10399v1.

[19] Hossienkhani, H., Najafi, A. and Azimi, N. (2014) Reconstruction of $f(R, T)$ Gravity in Anisotropic Cosmological Models of Accelerating Universe. Astrophysics and Space Science, 353, 311-317. https://doi.org/10.1007/s10509-014-2068-7

[20] Sundell, P. and Koivisto, T. (2015) Anisotropic Cosmology and Inflation from a Tilted Bianchi IX Model. Physical Review D, 92, 123529.

[21] Zubair, M., Azmat, H. and Noureen, I. (2017) Dynamical Analysis of Cylindrically Symmetric Anisotropic Sources in $f(R, T)$ gravity. The European Physical Journal C, 77, 169. https://doi.org/10.1140/epjc/s10052-017-4723-y

[22] Fayaz, V. and Hossienkhani, H., Zarei, Z., Ganji, M. and Azim, N. (2017) Anisotropic Universe and Reconstruction $f(R, T)$ Theory from Holographic Ricci Dark Energy. Canadian Journal of Physics, 95, 524-534. https://doi.org/10.1139/cjp-2016-0735

[23] Mishra, B., Tarai, S. and Tripathy, S.K. (2018) Dynamical Features of an Aniostropic Cosmological Model. Indian Journal of Physics. (In Press) 